Original paper

\title{
The FLUKA Monte Carlo code coupled with an OER model for biologically weighted dose calculations in proton therapy of hypoxic tumors
}

\author{
Tordis Johnsen Dahle ${ }^{\mathrm{a}, *}$, Espen Rusten ${ }^{\mathrm{b}, \mathrm{c}}$, Camilla Hanquist Stokkevåg ${ }^{\mathrm{a}, \mathrm{d}}$, Antti Silvoniemi ${ }^{\mathrm{e}, \mathrm{f}}$, \\ Andrea Mairani $^{g, h}$, Lars Fredrik Fjæra ${ }^{\mathrm{a}}$, Eivind Rørvik ${ }^{\mathrm{a}, \mathrm{c}}$, Helge Henjum ${ }^{\mathrm{a}}$, Pauliina Wright ${ }^{\mathrm{i}, \mathrm{j}}$, \\ Camilla Grindeland Boer ${ }^{\mathrm{d}}$, Sarita Forsback ${ }^{\mathrm{f}}$, Heikki Minn ${ }^{\mathrm{f}, \mathrm{i}}$, Eirik Malinen ${ }^{\mathrm{b}, \mathrm{c}}$, \\ Kristian Smeland Ytre-Hauge ${ }^{a}$
}

${ }^{a}$ Department of Physics and Technology, University of Bergen, Allégaten 55, 5007 Bergen, Norway

${ }^{\mathrm{b}}$ Department of Physics, University of Oslo, P.O. Box 1048 Blindern, 0316 Oslo, Norway

${ }^{\mathrm{c}}$ Department of Medical Physics, Oslo University Hospital, P.O. Box 4953 Nydalen, 0424 Oslo, Norway

${ }^{\mathrm{d}}$ Department of Oncology and Medical Physics, Haukeland University Hospital, Jonas Lies vei 65, 5021 Bergen, Norway

${ }^{\mathrm{e}}$ Department of Otorhinolaryngology - Head and Neck Surgery, Turku University Hospital, P.O. Box 52, 20521 Turku, Finland

${ }^{\mathrm{f}}$ Turku PET Centre, University of Turku, P.O. Box 52, 20521 Turku, Finland

${ }^{g}$ Centro Nazionale di Adroterapia Oncologica (CNAO Foundation), Str. Campeggi, 53, 27100 Pavia, Italy

${ }^{\mathrm{h}}$ Heidelberg Ion-Beam Therapy Center (HIT), Im Neuenheimer Feld 450, 69120 Heidelberg, Germany

${ }^{\mathrm{i}}$ Department of Oncology and Radiotherapy, Turku University Hospital, P.O. Box 52, 20521 Turku, Finland

${ }^{j}$ Department of Medical Physics, Turku University Hospital, P.O. Box 52, 20521 Turku, Finland

\section{A R T I C L E I N F O}

\section{Keywords:}

Hypoxia

Proton therapy

Oxygen enhancement ratio

Relative biological effectiveness

\begin{abstract}
A B S T R A C T
Introduction: The increased radioresistance of hypoxic cells compared to well-oxygenated cells is quantified by the oxygen enhancement ratio (OER). In this study we created a FLUKA Monte Carlo based tool for inclusion of both OER and relative biological effectiveness (RBE) in biologically weighted dose (ROWD) calculations in proton therapy and applied this to explore the impact of hypoxia.

Methods: The RBE-weighted dose was adapted for hypoxia by making RBE model parameters dependent on the OER, in addition to the linear energy transfer (LET). The OER depends on the partial oxygen pressure $\left(\mathrm{pO}_{2}\right)$ and LET. To demonstrate model performance, calculations were done with spread-out Bragg peaks (SOBP) in water phantoms with $\mathrm{pO}_{2}$ ranging from strongly hypoxic to normoxic $(0.01-30 \mathrm{mmHg}$ ) and with a head and neck cancer proton plan optimized with an RBE of 1.1 and $\mathrm{pO}_{2}$ estimated voxel-by-voxel using $\left[{ }^{18} \mathrm{~F}\right]$-EF5 PET. An RBE of 1.1 and the Rørvik RBE model were used for the ROWD calculations.

Results: The SOBP in water had decreasing ROWD with decreasing $\mathrm{pO}_{2}$. In the plans accounting for oxygenation, the median target doses were approximately a factor 1.1 lower than the corresponding plans which did not consider the OER. Hypoxia adapted target ROWDs were considerably more heterogeneous than the $\mathrm{RBE}_{1.1^{-}}$ weighted doses.

Conclusion: We realized a Monte Carlo based tool for calculating the ROWD. Read-in of patient $\mathrm{pO}_{2}$ and estimation of ROWD with flexibility in choice of RBE model was achieved, giving a tool that may be useful in future clinical applications of hypoxia-guided particle therapy.
\end{abstract}

\section{Introduction}

Cells with partial oxygen pressure $\left(\mathrm{pO}_{2}\right)$ lower than $8-10 \mathrm{mmHg}$ are generally termed hypoxic [1] and are more radioresistant than cells with normal oxygen levels $\left(\mathrm{pO}_{2}\right.$ up to around $100 \mathrm{mmHg}$ [1]). This effect may be quantified by the oxygen enhancement ratio (OER), which is the ratio of the dose at a given oxygen pressure to that at a normal oxygen pressure producing the same biological effect. The OER is comparable for photons and protons, but decreases at low proton energies where the linear energy transfer (LET) is higher [2]. This effect is more pronounced for heavier particles such as carbon ions. In radiotherapy treatment planning, the local dose should ideally reflect the oxygen levels to minimize radiation exposure while remaining effective. Furthermore, the relative biological effectiveness (RBE) is

\footnotetext{
* Corresponding author.

E-mail address: tordis.dahle@uib.no (T.J. Dahle).
} 
applied to transfer clinical treatment protocols from photon therapy to proton therapy. This is currently implemented by delivering a homogeneous RBE-weighted dose to the target, with the RBE set to 1.1 [3]. However, the RBE is known to vary and several RBE models at normoxic conditions have been proposed [4-7]. To account for the varying oxygen levels and the variable RBE in particle therapy, a common framework for combing the effects in RBE and OER weighted dose (ROWD) calculations could improve the patient treatment outcome.

A common method of measuring the $\mathrm{pO}_{2}$ in vivo is by invasive oxygen-sensitive electrodes. However, such methods are limited to accessible tissues and does not give a full three-dimensional representation of the $\mathrm{pO}_{2}$ levels in the patient [8]. With functional imaging such as positron emission tomography (PET) or magnetic resonance imaging, information about the oxygen level can be obtained on a voxel-by-voxel basis [9]. Several methods for depicting hypoxia with functional imaging have been proposed, and PET is currently the preferred modality when studying hypoxia in clinical settings [10]. Among the tracers in clinical use, two hypoxia radiotracers for PET are ${ }^{18}$ F-fluoromisonisazole ( $\left[{ }^{18} \mathrm{~F}\right]$-FMISO) and ${ }^{18} \mathrm{~F}$-labeled 2-(2-nitro- ${ }^{1} \mathrm{H}$-imidazol-1yl)-N-(2,2,3,3,3-pentafluoropropyl)-acetamide ( $\left[{ }^{18} \mathrm{~F}\right]$-EF5). While $\left[{ }^{18} \mathrm{~F}\right]$-FMISO is the most actively investigated hypoxia PET tracer, $\left[{ }^{18} \mathrm{~F}\right]$ EF5 has the advantage that the non-labeled form of this compound has been widely used to study hypoxia in clinical and preclinical settings. Moreover, the hypoxia-avidity of this tracer has been demonstrated convincingly [11-13]. Also, EF5 binding in hypoxic head-and-neck squamous cell cancer is rather well established and has been shown to relate with poor outcome in photon radiochemotherapy $[14,15]$.

Monte Carlo (MC) simulations are frequently used to provide estimates of the RBE-weighted dose in proton and carbon ion therapy, and several RBE models which do not account for hypoxia have already been implemented in the FLUKA MC code $[16,17]$. This includes the local effect model (LEM) [18,19] and the microdosimetric kinetic model (MKM) [20,21], whereas several methods for accounting for both RBE and hypoxia in particle therapy treatment planning have been suggested [22-25]. Scifoni et al. [23] and Tinganelli et al. [25] described a ROWD optimization algorithm incorporating OER (through a semiempirical model) and RBE (using LEM IV), and implemented this into the analytical treatment planning system (TPS) TRiP98 [26,27]. They applied an OER model which was intended for particle therapy with heavier ions like carbon and oxygen. For protons, the same ROWD estimation method can be applied, however, an OER model developed specifically for protons would be more suitable, as the universal OER models may overestimate the OER for protons [2]. Wenzl and Wilkens [28] developed an OER model with parameters which depend on the LET and the $\mathrm{pO}_{2}$, which was based on experimental data from several types of particles, including, but not limited to, protons and carbon ions. To our knowledge, none of the currently available OER models have parameters based on proton data only, although Strigari et al. [24] included a particle dependency in their calculation of the OER. In this study we developed a method for recalculating treatment plans with a ROWD, with flexibility regarding the choice of RBE model, and implemented the method in the FLUKA MC code. This will indicate potential areas of lower tumor dose effect in a volume with varying degree of hypoxia. Including the method in the dose optimization process would then enable delivering a homogeneous ROWD, i.e. equal biological effect across regions with different oxygen levels.

\section{Materials and methods}

\subsection{Estimation of oxygen levels in a patient}

The patient oxygen levels, given as $\mathrm{pO}_{2}$, were estimated using PET images of head and neck cancer patients with $\left[{ }^{18} \mathrm{~F}\right]$-EF5 as hypoxia tracer. The PET images were acquired between 2013 and 2016 using a GE D690 PET/CT scanner (General Electric Medical Systems, Milwaukee, WI, USA) at Turku University Hospital (Finland) [29]. The relationship between PET uptake and $\mathrm{pO}_{2}$ was estimated applying the following equation:

Uptake $=A-\frac{B \cdot \mathrm{pO}_{2}}{C+\mathrm{pO}_{2}}$,

where $A, B$ and $C$ are reaction-specific parameters [30]. We estimated these parameters for $\left[{ }^{18} \mathrm{~F}\right]$-EF5 PET by non-linear least square curve fit of the uptake data from PET images of six head and neck cancer patients in organs with $\mathrm{pO}_{2}$ values reported in literature, see Appendix A for details. The PET uptake was normalized to mean muscle uptake, as a tumor-to-muscle $(\mathrm{T} / \mathrm{M})$ ratio of 1.5 at $3 \mathrm{~h}$ from injection has been found to be an appropriate threshold for determining clinical significant hypoxia in $\left[{ }^{18} \mathrm{~F}\right]$-EF5 PET images [13].

\subsection{Calculations of oxygen enhancement ratio}

The OER was calculated applying a version of the model by Wenzl and Wilkens [28], modified by obtaining the model coefficients by regression to in vitro proton data only. The Wenzl and Wilkens [28] OER model calculates the survival fraction of cells, $S$, after ion irradiation according to the linear quadratic (LQ) model [31]:

$-\ln (S)=\alpha D+\beta D^{2}$

where $D$ is the physical dose. $\alpha$ and $\beta$ are the radiosensitivity parameters, defined by Wenzl and Wilkens [28] as follows:

$\alpha(L, p)=\frac{\left(a_{1}+a_{2} \cdot L\right) \cdot p+\left(a_{3}+a_{4} \cdot L\right) \cdot K}{p+K}$,

$\sqrt{\beta(p)}=\frac{b_{1} \cdot p+b_{2} \cdot K}{p+K}$,

where $L$ is the dose-averaged LET $\left(\operatorname{LET}_{\mathrm{d}}\right), p$ is the $\mathrm{pO}_{2}$, and $K$ is set to $3 \mathrm{mmHg}$ [28]. By non-linear least square curve fit of in vitro proton data (Fig. 1 and Table B.1 in Appendix B) the model parameters were found to be: $a_{1}=0.10 \mathrm{~Gy}^{-1}, a_{2}=0.0010 \mu \mathrm{m} /(\mathrm{Gy} \cdot \mathrm{keV}), a_{3}=0.010 \mathrm{~Gy}^{-1}$, $a_{4}=0.0100 \mu \mathrm{m} /\left(\mathrm{Gy} \cdot \mathrm{keV}\right.$ ), $b_{1}=0.765 \mathrm{~Gy}^{-1}$ and $b_{2}=0.273 \mathrm{~Gy}^{-1}$ (see Appendix B for details). From this, the OER can be calculated:

$\operatorname{OER}\left(L, p_{h}\right)=\frac{\sqrt{\alpha^{2}\left(L, p_{h}\right)-4 \beta\left(p_{h}\right) \cdot \ln (S)}-\alpha\left(L, p_{h}\right)}{\sqrt{\alpha^{2}\left(L, p_{a}\right)-4 \beta\left(p_{a}\right) \cdot \ln (S)}-\alpha\left(L, p_{a}\right)} \cdot \frac{\beta\left(p_{a}\right)}{\beta\left(p_{h}\right)}$,

where $p_{h}$ and $p_{a}$ are the oxygen pressure at the hypoxic and aerobic (or normoxic) conditions, respectively. In our implementation $p_{h}$ was

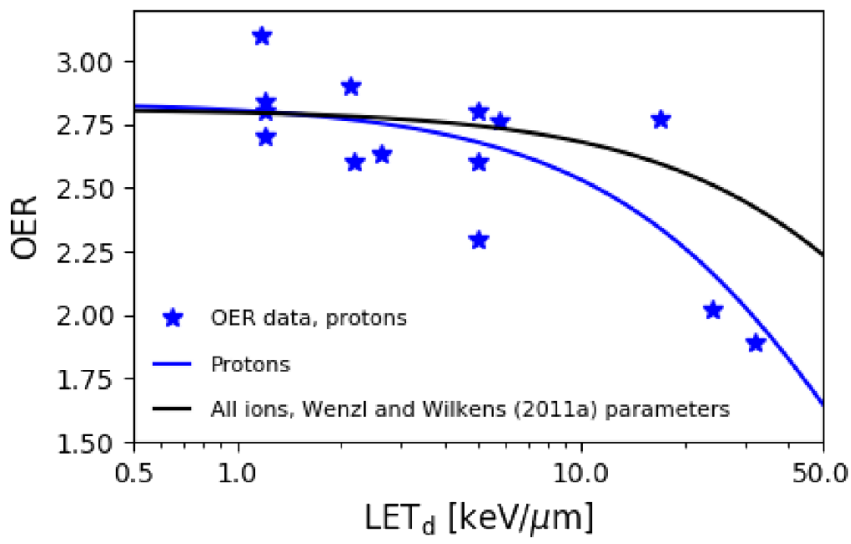

Fig. 1. In vitro proton data at $10 \%$ cell survival (stars) and OER calculations done according to Eq. (5), with $p_{a}=160 \mathrm{mmHg}$ and $p_{h}=0.01 \mathrm{mmHg}$ (lines). The blue line is obtained by non-linear least square curve fit to the proton data (stars), while the black line applies parameters given by Wenzl and Wilkens [28] (given in Table B.2. Appendix B), obtained by fitting to data from several different ions. See Appendix B for details on the proton in vitro data. (For interpretation of the references to colour in this figure legend, the reader is referred to the web version of this article.) 
obtained from the PET image and we set $p_{a}$ equal to $30 \mathrm{mmHg}$ as this is a typical $\mathrm{pO}_{2}$ value for tissues [28]. This value for $p_{a}$ is lower than what is given in Fig. 1. Fig. 1 applies the aerobic condition in in vitro experiments, which is generally fixed to $160 \mathrm{mmHg}$ and higher than what is normally found in vivo [28]. As the model parameters are based on a survival fraction of $10 \%$, we also set the survival level to $10 \%$ in our calculations.

\subsection{RBE-weighted dose calculations for normoxic conditions}

To adapt the RBE-weighted dose for hypoxia, a short description of RBE calculations for normoxic conditions is needed. For a more thorough description, see Rørvik et al. [32]. In general, the RBE is calculated from the LQ model as follows:

$\mathrm{RBE}=\frac{1}{D_{p}}\left(\sqrt{\left(\frac{\alpha_{x}}{2 \beta_{x}}\right)^{2}+\frac{\left(\alpha_{a} D_{p}+\beta_{a} D_{p}^{2}\right)}{\beta_{x}}}-\frac{\alpha_{x}}{2 \beta_{x}}\right)$,

where $D_{p}$ is the physical proton dose deposited per fraction and $\alpha_{a}, \beta_{a}$, $\alpha_{x}$ and $\beta_{x}$ are the normoxic proton and photon radiosensitivity parameters, respectively. The various RBE models differ in how these radiosensitivity parameters are defined [32]. We applied two methods to calculate the RBE, as described below.

In the first method, the RBE-weighted dose $\left(D_{\mathrm{RBE} 1.1}\right)$ was calculated using a constant RBE of 1.1. To achieve this using Eq. (6), which is required to later include the OER in the calculations, we used the same radiosensitivity parameters for photons and protons, i.e. $\alpha_{a}=\alpha_{x}$ and $\beta_{a}=\beta_{x}$. This would give the same survival curve for protons and photons, and thus an RBE of 1 . The $D_{\mathrm{RBE} 1.1}$ is then given by Eq. (6) multiplied by 1.1 and total physical dose, $D$ :

$D_{\mathrm{RBE} 1.1}=1.1 \cdot \frac{D}{D_{p}}\left(\sqrt{\left(\frac{\alpha_{x}}{2 \beta_{x}}\right)^{2}+\frac{\left(\alpha_{a} D_{p}+\beta_{a} D_{p}^{2}\right)}{\beta_{x}}}-\frac{\alpha_{x}}{2 \beta_{x}}\right)$,

where $\alpha_{a}=\alpha_{x}$ and $\beta_{a}=\beta_{x}$, and are given as in the previously described OER model (Eqs. (3) and (4)), with a $\mathrm{pO}_{2}$ value set to $30 \mathrm{mmHg}$ and a constant $\mathrm{LET}_{\mathrm{d}}$ of $0.3 \mathrm{keV} / \mu \mathrm{m}$.

In the second method, the RBE-weighted dose ( $\left.D_{\mathrm{ROR}}\right)$ was estimated applying the variable RBE model by Rørvik et al. [5] (see Appendix C for details). We applied an $(\alpha / \beta)_{x}=2$ Gy for all structures. However, published $(\alpha / \beta)_{x}$ ratios for head and neck cancer vary significantly, and when applying the tool for further testing, a larger range of $(\alpha / \beta)_{x}$ ratios should be applied [33]. As the goal here was to demonstrate the method, we chose a $(\alpha / \beta)_{x}$ ratio representing organs at risk (OARs) and late responding target volumes which have low $(\alpha / \beta)_{x}$ values [32].

\subsection{RBE and OER weighted dose calculations}

To account for hypoxia in the calculations of ROWD, we have, similar to Scifoni et al. [23] and Tinganelli et al. [25], replaced the normoxic proton response parameters in Eq. (6) with hypoxic proton response parameters which vary with the OER:

$\alpha_{h}=\alpha_{a} / \operatorname{OER}\left(L, p_{h}\right)$,

$\beta_{h}=\beta_{a} / \operatorname{OER}^{2}\left(L, p_{h}\right)$,

where the OER is the OER at $10 \%$ cell survival calculated according to Eq. (5). This ROWD model allows us to apply $\alpha_{a}, \beta_{a}, \alpha_{x}$ and $\beta_{x}$ as defined in most of the existing RBE models derived from normoxic proton and photon in vitro data and then estimate $\alpha_{h}$ and $\beta_{h}$ applying Eqs. (8) and (9). The ROWD accounting for hypoxia is then given as follows:

$D_{\mathrm{OER}, \mathrm{RBE}}=\frac{D}{D_{p}}\left(\sqrt{\left(\frac{\alpha_{x}}{2 \beta_{x}}\right)^{2}+\frac{\alpha_{h} D_{p}+\beta_{h} D_{p}^{2}}{\beta_{x}}}-\frac{\alpha_{x}}{2 \beta_{x}}\right)$, where $D$ is the total deposited physical dose and $D_{p}$ is the physical dose deposited by protons only. While this ROWD model will give an isoeffective dose accounting for hypoxia, we called it $D_{\mathrm{OER}, \mathrm{RBE}}$ to emphasize that it is not directly the RBE-weighted dose, as only the proton parameters take hypoxia into account and not the photon parameters. The $\alpha_{x}$ and $\beta_{x}$ parameters are always the normoxic photon radiosensitivity parameters.

The $D_{\mathrm{RBE} 1.1}$ and the $D_{\mathrm{ROR}}$ models were adapted to variable oxygen levels by changing their respective $\alpha_{a}$ and $\beta_{a}$ parameters to $\alpha_{h}$ and $\beta_{h}$ according to Eqs. (8) and (9), and keeping $\alpha_{x}$ and $\beta_{x}$ as defined in each model. To distinguish the models adapted for hypoxia and not adapted for hypoxia, the models adapted for hypoxia will hereafter be referred to as $D_{\mathrm{OER}, \mathrm{RBE} 1.1}$ and $D_{\mathrm{OER}, \mathrm{ROR}}$.

\subsection{Implementation of the method in FLUKA}

Our in-house made system [34] for recalculating treatment plans in FLUKA was modified to include the hypoxia inclusive ROWD calculation method. This tool translates DICOM information from a treatment plan generated in an analytical TPS to files readable by FLUKA. This includes information on the radiation beam and scrips translating the CT image into FLUKA geometry. When simulating a treatment plan, we scored $\mathrm{LET}_{\mathrm{d}}, \mathrm{pO}_{2}$, physical dose $D, \alpha_{a} D$ and $\sqrt{\beta_{a}} D$ in FLUKA. The OER was then calculated in Python according to Eq. (5), using $\mathrm{LET}_{\mathrm{d}}, \mathrm{pO}_{2}$ and $D$ from FLUKA, and the ROWD was subsequently calculated using Eq. (10).

$\operatorname{LET}_{\mathrm{d}}, \alpha_{a} D, \sqrt{\beta_{a}} D$ and $\mathrm{pO}_{2}$ were scored using the FLUKA subroutine fluscw (FLUence Scoring Weight). LET $_{\mathrm{d}}$ was here estimated applying the same approach as in Fjæra et al. [34], i.e. using the following equation:

$\operatorname{LET}_{\mathrm{d}}=\frac{\sum_{i} \int \Phi_{i}(E)\left(\operatorname{LET}_{i}(E)\right)^{2} d E}{\sum_{i} \int \Phi_{i}(E)\left(\operatorname{LET}_{i}(E)\right) d E}$,

where $\Phi_{i}(E)$ is the fluence of particle species $i$ with kinetic energy $E$ and $\operatorname{LET}_{i}(E)$ is the unrestricted LET for the same kinetic energy. To score $\mathrm{pO}_{2}$, we implemented a method for importing estimated $\mathrm{pO}_{2}$ values in a patient geometry on a voxel-by-voxel basis in FLUKA using fluscw. This subroutine reads the $\mathrm{pO}_{2}$ values from a table, created from the PET images; the coordinate system of the PET image was converted into FLUKA coordinates, and for each particle position, the corresponding $\mathrm{pO}_{2}$ value from the PET image was given. Subsequently, $\mathrm{pO}_{2}$ times dose to water and dose to water were scored in FLUKA. Then, $\mathrm{pO}_{2}$ was given by the quotient of the two scored quantities. To achieve the needed accuracy of the scored values without increasing the simulation time significantly, we set the fraction of the kinetic energy to be lost in a simulation step to 0.0125 , which was $25 \%$ of the default value when applying the FLUKA HADROTHErapy defaults.

\subsection{Treatment plans}

Two spread-out Bragg peak (SOBP) scenarios in water and a treatment plan for head and neck cancer were used to test the MC tool and investigate the impact of hypoxia on ROWD estimations. The treatment plans were optimized using an RBE of 1.1 in the Eclipse (Varian Medical Systems, Palo Alto, CA, USA) TPS, not accounting for hypoxia, and then recalculated to account for hypoxia and RBE using our FLUKA based tool. For all simulations, the number of simulated primary particles was chosen to have a voxel mean statistical uncertainty $<2 \%$.

The first SOBP scenario had the same dimensions as the $\mathrm{pO}_{2}$ table, with scoring voxels of $\left(1.95 \times 1.95 \times 2.4 \mathrm{~mm}^{3}\right)$. This SOBP scenario was only used to check the FLUKA estimation of $\mathrm{pO}_{2}$ values. The second SOBP scenario (starting at depth $8 \mathrm{~cm}$ and with size $4 \times 4 \times 4 \mathrm{~cm}^{3}$ ) was optimized to an $D_{\mathrm{RBE} 1.1}$ dose of $2 \mathrm{~Gy}(\mathrm{RBE})$ and applied in four simulated water phantoms. Each simulated water phantom had homogeneously distributed $\mathrm{pO}_{2}$ values, with each of the four phantoms 
having a given $\mathrm{pO}_{2}: 0.01 \mathrm{mmHg}, 0.5 \mathrm{mmHg}, 3.0 \mathrm{mmHg}$ or $30.0 \mathrm{mmHg}$. The simulated phantoms had scoring voxels of same size as in the TPS $\left(2 \times 2 \times 2 \mathrm{~mm}^{3}\right)$.

The head and neck cancer patient was originally treated at Turku University Hospital with chemoradiotherapy [29], and the PET images of this patient were included in creating the PET uptake to $\mathrm{pO}_{2}$ conversion curve (Appendix A). For this study, we created a proton treatment plan for the patient, optimized (using an RBE of 1.1) in the Eclipse TPS with prescribed dose of $70 \mathrm{~Gy}$ (RBE) in 35 fractions to the planning target volume (PTV). $\mathrm{pO}_{2}$ values of the patient were estimated from $\left[{ }^{18} \mathrm{~F}\right]$-EF5 PET/CT images of the patient, and the FLUKA scoring was done using the same grid and voxel size $\left(1.95 \times 1.95 \times 2.50 \mathrm{~mm}^{3}\right)$ as used during the TPS optimization.

\section{Results}

\subsection{FLUKA readout of $\mathrm{pO}_{2}$}

The generated $\mathrm{pO}_{2}$ map was successfully read by FLUKA, as shown in Fig. D. 1 in Appendix D. The process of importing patient $\mathrm{pO}_{2}$ data in FLUKA started with a $\left[{ }^{18} \mathrm{~F}\right]$-EF5 PET image (Fig. D.1a). The conversion from PET uptake to $\mathrm{pO}_{2}$ was done applying Eq. (1) with reaction-specific parameters found to be $A=2.60, B=1.98$ and $C=2.5 \mathrm{mmHg}$, see Appendix A for details. The estimated $\mathrm{pO}_{2}$ table (Fig. D.1b) corresponding to the PET image showed areas of low $\mathrm{pO}_{2}$ values in the PTV, with minimum, mean and maximum $\mathrm{pO}_{2}$ in the PTV of 2.2, 16.8 and $60.0 \mathrm{mmHg}$, respectively. This was also reproduced by the FLUKA imported $\mathrm{pO}_{2}$ values (Fig. D.1c).

\subsection{Water phantom}

Compared to $D_{\mathrm{RBE} 1.1}$, the ROWD accounting for hypoxia decreased with decreasing $\mathrm{pO}_{2}$ values, as seen in Fig. 2. The decrease rate typically followed the factors corresponding to the OER values for respective oxygenation levels in Fig. 2. This shows that the proton irradiation is predicted to be less effective at low oxygen levels, as expected from the definition of the OER. At $30 \mathrm{mmHg}$, which is a normoxic condition, the $D_{\mathrm{OER}, \mathrm{RBE} 1.1}$ dose was equal to the prescribed dose, as expected. Applying the $D_{\mathrm{OER}, \mathrm{ROR}}$ model, however, an RBE of 1.09 was observed at the beam entrance, increasing to 1.38 at the distal end of the beam, at normoxic condition. For all oxygen levels, the OER value was seen to be mostly constant along the beam path, with a slight decrease at high $\mathrm{LET}_{\mathrm{d}}$ values. This decrease was most significant at low oxygen levels. At $0.01 \mathrm{mmHg}$, which is an extremely hypoxic condition, the OER value started at 2.7 for low $\mathrm{LET}_{\mathrm{d}}$ values and decreased to 2.3 at the end of the beam range.

\subsection{Patient case}

The $\mathrm{pO}_{2}$ map of the head and neck cancer patient is shown in Fig. 3a. The treatment plan had $\mathrm{LET}_{\mathrm{d}}$ values in the range of 1.2-4.3 keV/ $\mu \mathrm{m}$ in the PTV (Fig. 3b). The dose volume histograms (DVHs) of the $D_{\mathrm{RBE} 1.1}, D_{\mathrm{ROR}}, D_{\mathrm{OER}, \mathrm{RBE} 1.1}$ and $D_{\mathrm{OER}, \mathrm{ROR}}$ are shown in Fig. 3c. The $D_{\text {ROR }}$ model gave the largest dose to the PTV, and the $D_{\text {RBE1.1 }}$ the second largest dose, except in $18 \%$ of the volume where the $D_{\text {OER,ROR }}$ had larger dose, as $D_{\text {OER,ROR }}$ gives the same dose as the $D_{\text {ROR }}$ model in the normoxic parts of the PTV. The normoxic RBE-weighted doses ( $D_{\mathrm{RBE} 1.1}$ and $D_{\mathrm{ROR}}$ ) had the steepest DVHs, which was also reflected in the dose distributions shown in Fig. 3d-f, where a homogenous $D_{\mathrm{RBE} 1.1}$ to the PTV was observed, while the ROWDs accounting for hypoxia had areas with clearly lower dose. The corresponding $D_{\mathrm{ROR}}$ distribution is given in Fig. 4, showing also a mainly homogeneous dose distribution to the PTV.

The median target dose $\left(D_{50 \%}\right)$, with the range from the near minimum target dose $\left(D_{98 \%}\right)$ to the near maximum target dose $\left(D_{2 \%}\right)$ in parenthesis, was $69.9 \mathrm{~Gy}(\mathrm{RBE})$ (62.8-74.3 Gy(RBE)), $62.6 \mathrm{~Gy}(\mathrm{RBE})$ (52.6-73.9 Gy(RBE)), 74.0 Gy(RBE) (65.3-78.9 Gy(RBE)) and $66.5 \mathrm{~Gy}$ (RBE) (56.8-78.9 Gy(RBE)) when estimated applying $D_{\text {RBE1.1, }}$ $D_{\text {OER,RBE1.1 }}, D_{\text {ROR }}$ and $D_{\text {OER,ROR }}$ respectively. The dose difference between $D_{\text {RBE1.1 }}$ and $D_{\mathrm{OER}, \mathrm{RBE} 1.1}$ is given in Fig. $3 \mathrm{~g}$, showing even clearer that low oxygen levels result in lower biological effect of the radiation, as expected. This is also seen in Fig. 4 , where the $D_{\mathrm{ROR}}-D_{\mathrm{OER}, \mathrm{ROR}}$ dose difference is given, showing that the differences $D_{\mathrm{ROR}}-D_{\mathrm{OER}, \mathrm{ROR}}$ and $D_{\text {RBE1.1 }}-D_{\text {OER,RBE1.1 }}$ are comparable. The similar shapes of the dose difference can be attributed to the approximately homogeneous $\operatorname{LET}_{\mathrm{d}}$ and physical dose distribution in the target.

\section{Discussion}

In this study we developed a tool for calculating the ROWD in hypoxic conditions for protons, by coupling the FLUKA MC code with a ROWD calculation model. The tool was tested with simple treatment plans for homogeneous water phantoms with several oxygen levels as well as on a head and neck cancer patient plan with $\mathrm{pO}_{2}$ estimated from $\left[{ }^{18} \mathrm{~F}\right]$-EF5 PET images. Applying a method based on the LQ model has several benefits, one being that the RBE effects are also directly included in the calculations. Such calculations may become useful in future clinical applications of hypoxia-guided particle therapy.

Scifoni et al. [23] and Tinganelli et al. [25] accounted for hypoxia applying the same approach as in the current work, by using the LEM and a dedicated OER model implemented in the TRiP98 TPS, which facilitated RBE and OER driven optimization (kill painting). Wouters and Brown [35], and later Malinen et al. [36], also applied this formalism to estimate hypoxic $\alpha$ and $\beta$ values from normoxic $\alpha$ and $\beta$
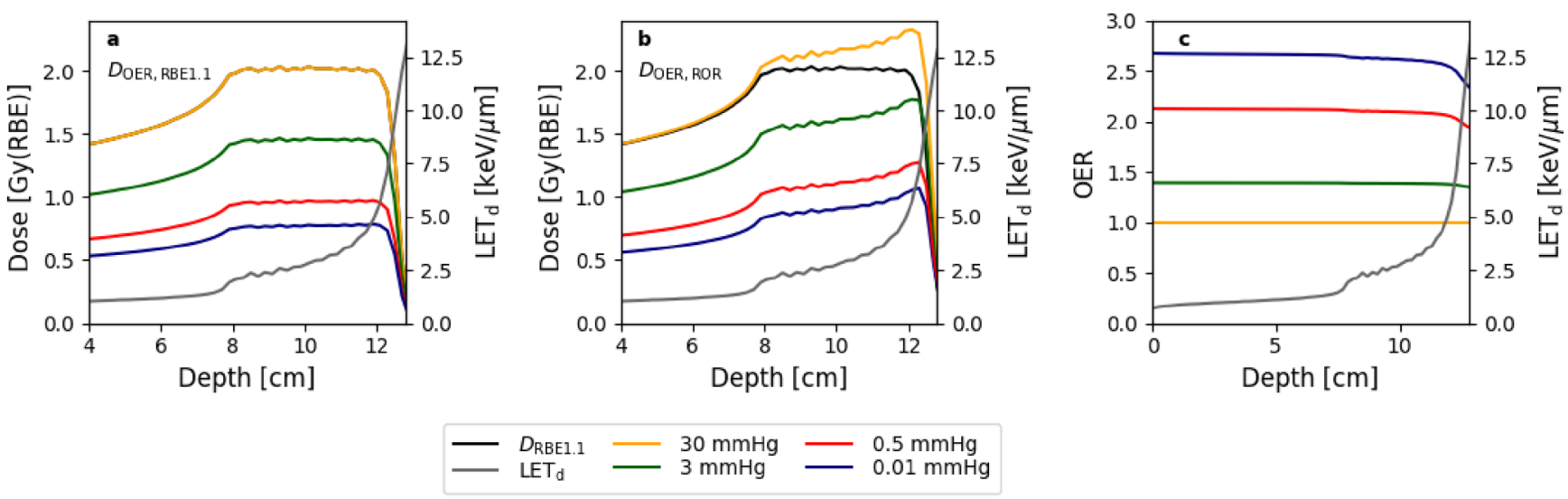

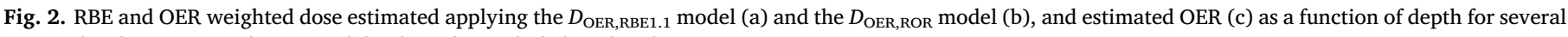
oxygen levels. $\mathrm{LET}_{\mathrm{d}}$ as a function of depth is also included in the plots. 

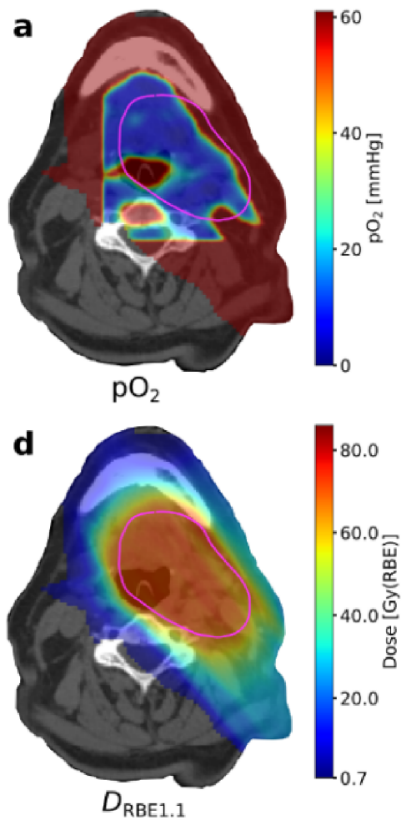
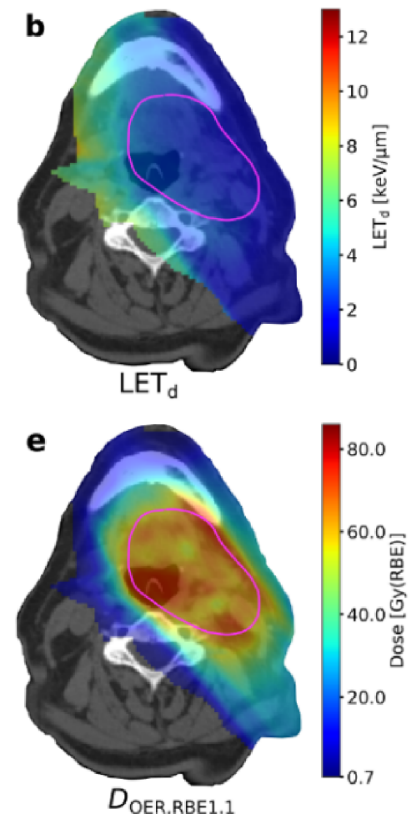
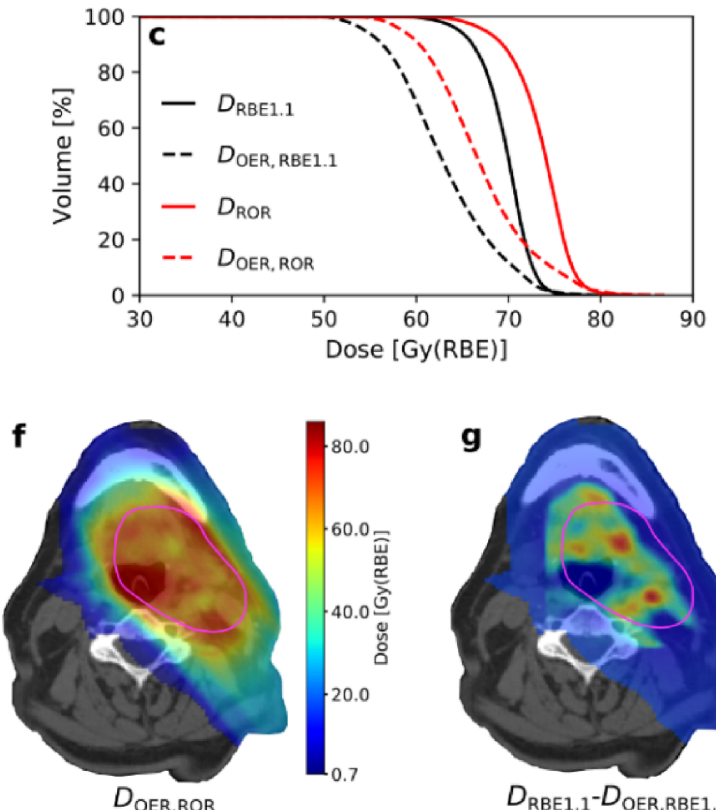

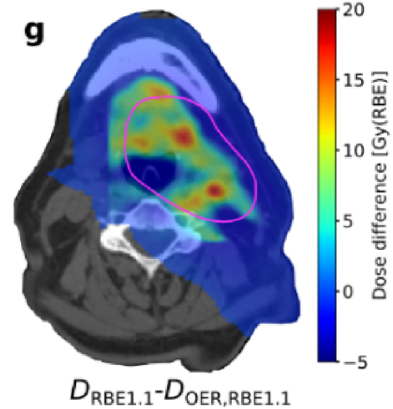

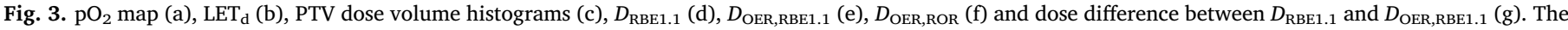

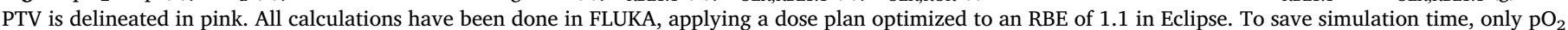

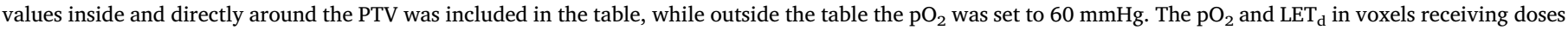
below $0.7 \mathrm{~Gy}(\mathrm{RBE})$ according to the $D_{\mathrm{RBE} 1.1}$ dose (1\% of prescription dose) is set transparent.
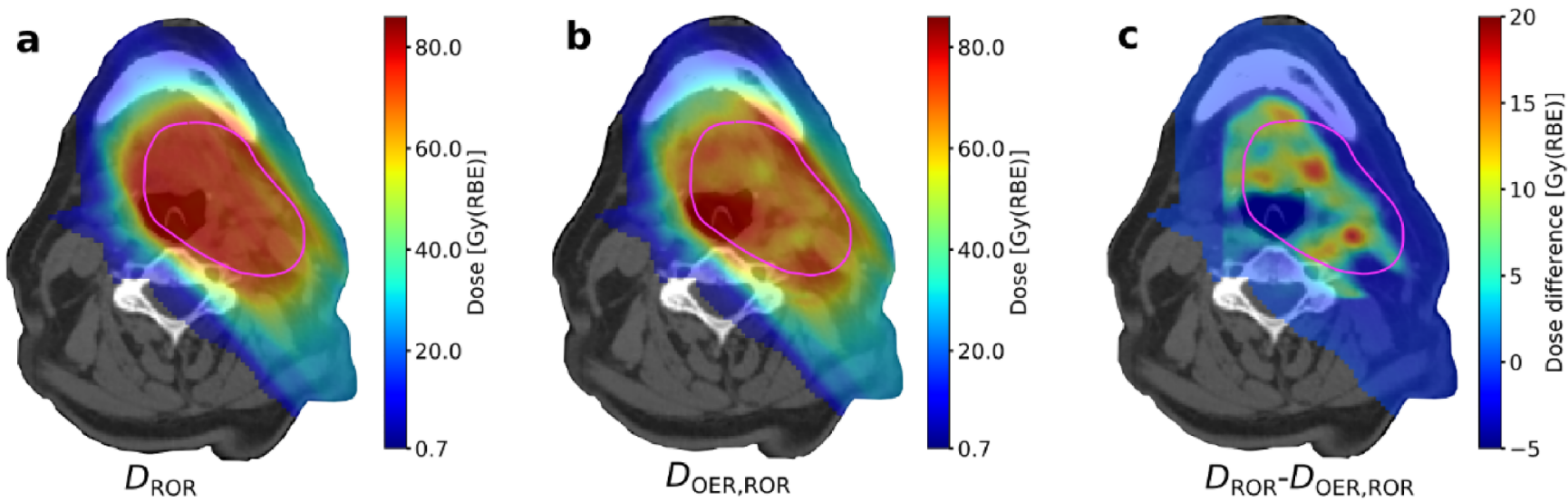

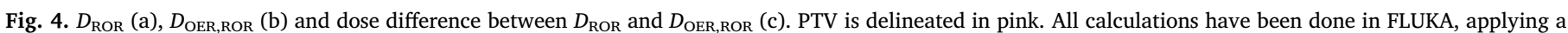
dose plan optimized to an RBE 1.1 in Eclipse.

parameters. Using this approach, the physical dose is weighted by the inverse of the OER before calculating the RBE. Due to the dose dependency of a variable RBE, the RBE will therefore be slightly dependent on the OER. The estimated ROWD is therefore not equal to the RBE-weighted dose divided by the OER, but the difference is small.

We applied our tool using the variable RBE model by Rørvik et al. [5], in addition to a constant RBE of 1.1. The tool could also be applied on most other normoxic proton RBE models, as well as RBE models for heavier ions, which follow the general RBE equation (Equation (6)). The OER model used in this study was based on the model by Wenzl and Wilkens [28], but with model parameters estimated from proton in vitro data only, as the OER has been seen to decrease significantly faster with the $\operatorname{LET}_{\mathrm{d}}$ when applying low-LET irradiation compared to high-LET irradiation, especially towards the end of the particle range [2]. Strigari et al. [24] observed this same trend, i.e. the lighter the particles the faster the OER decreases with increasing LET, when computing OER vs. LET curves for different particle types. This was also observed in our study, as the OER decreased faster when applying proton parameters than when applying parameters based on the data for several ion types
(Fig. 1). When applying the tool to high-LET radiation, e.g. carbon ions, the OER model parameters must be adjusted to data taken from heavier ions, for instance using the parameters given by Wenzl and Wilkens [28].

The Wenzl and Wilkens [28] OER model has been seen to deviate from other existing OER models, resulting in lower OER for low LET radiation compared to the models by Scifoni et al. [23], Strigari et al. [24] and Antonovic et al. [37]. However, this may be due to the optimization of the model parameters [24]. For instance, using the model parameters in the Wenzl and Wilkens [28] OER model one assumes that all hypoxic in vitro data has a controlled $\mathrm{pO}_{2}$ of $0.01 \mathrm{mmHg}$. To tune the existing OER models and judge which one is best, more experimental data is required. To reduce the uncertainty in experimental OER data, it is necessary to estimate LET values accurately and consistently between experiments, preferably using Monte Carlo simulations of the irradiation setup $[38,39]$. In addition, as the OER is dependent on the tissue type, there may be differences between the clinical OER and the in vitro OER, which may influence the results since the OER model parameters are generally based on in vitro data. However, Wenzl and Wilkens [28] 
showed that there was generally good agreement between their OER model and results from preclinical and clinical studies (mainly performed applying photon irradiation). We therefore decided to use this model in our calculations, although uncertainties in the OER model must be acknowledged.

In the current OER model, the parameters were designed for a cell survival of $10 \%$, ignoring a possible dose dependency. Wenzl and Wilkens [40] studied this dependency and found that the OER did not vary significantly with the dose, especially at high LET values and high $\mathrm{pO}_{2}$ values. At lower LET values there was a clear dose dependency, but this was most pronounced for extremely hypoxic cells with lower $\mathrm{pO}_{2}$ than what was seen in our patient case. The dose dependency also differed significantly between different cell lines. We therefore chose to maintain the survival level at $10 \%$ in our calculations, even though this may be a minor shortcoming. To extend the model to other survival levels, more experimental data is needed to fit the model parameters. Scifoni et al. [23] and Tinganelli et al. [25] did also ignore the dose dependency of the OER, although justified as their method was applied to high LET radiation.

The MC tool presented in this study depends on estimations of the $\mathrm{pO}_{2}$ levels in a patient on a voxel-by-voxel basis. As our main objective was to demonstrate the tool performance, the $\mathrm{pO}_{2}$ estimates should be regarded as an approximation in order to provide an indication of how $\mathrm{pO}_{2}$ values will influence clinical scenarios. Toma-Dasu et al. [30] assumed that PET uptake as a function of $\mathrm{pO}_{2}$ follows a sigmoid shaped curve, as the inhibition of chemical reactions typically follows this form. They showed their method for $\left[{ }^{18} \mathrm{~F}\right]$-FMISO PET and $\left[{ }^{18} \mathrm{~F}\right]$-FETA PET. While these tracers are, just like $\left[{ }^{18} \mathrm{~F}\right]$-EF5, nitroimidazole tracers, it is not certain that the conversion from $\left[{ }^{18} \mathrm{~F}\right]$-EF5 uptake to $\mathrm{pO}_{2}$ follows exactly this form, as different radiotracers have different uptake properties and with that different capabilities of imaging $\mathrm{pO}_{2}$ [30]. This method has also been applied for other PET tracers, including $\left[{ }^{18} \mathrm{~F}\right]$ HX4 PET [41]. While uncertainties are expected, the $\mathrm{pO}_{2}$ conversion curve was only applied to the tumor area and its proximity to save simulation time, and the $\mathrm{pO}_{2}$ of the remaining tissue was set to $60 \mathrm{mmHg}$. Thus, uncertainties in $\mathrm{pO}_{2}$ estimation will not have significant impact on the dose to the normal tissue. Also, the lowest $\mathrm{pO}_{2}$ value in the tumor applied in this study was estimated to be $2.2 \mathrm{mmHg}$, which corresponds to an OER of approximately 1.6. If the conversion curve (Appendix A, Fig. A.1) was less steep, as the data may suggest, this OER value would be an underestimation. An underestimation may not give high enough dose escalation to the tumor; however, it will be safer for the normal tissue.

The method to account for hypoxia in our study is, as mentioned, similar to the one on which the kill painting algorithm is based $[23,25]$. Kill painting was first proved experimentally, using cell exposure chambers which allow irradiating of cell cultures under defined oxygenation conditions [23,25]. Later, kill painting was applied in a treatment planning study with TRiP98 and an artificially generated $\left[{ }^{18} \mathrm{~F}\right]$-FMISO-PET derived hypoxia map of a patient [42]. In addition to this method, hypoxia has earlier also been accounted for in treatment planning studies applying dose and/or LET painting, i.e. where the hypoxic volumes receive either increased dose and/or increased LET values, respectively [43-45]. Malinen and Søvik [43] found that hypoxia dose painting appeared to be especially attractive for protons, while both LET and dose painting has the potential to increase the tumor control probability for heavier ions like lithium and carbon. Chang et al. [46] also studied dose painting and showed that dose painting in conventional radiotherapy based on $\left[{ }^{18} \mathrm{~F}\right]$-FMISO PET is technically feasible and has clear benefits in terms of increasing the tumor control probability. Kjellsson Lindblom et al. [41] showed, in addition, that dose painting in photon therapy based on $\left[{ }^{18} \mathrm{~F}\right]$-HX4 PET appears feasible in non-small cell lung cancer. Our tool does not yet include optimization of treatment plans based on the oxygenation levels; however, future work includes implementing the method in a FLUKA Monte Carlo based treatment planning system.

\section{Conclusion}

The Monte Carlo based tool introduced in this study facilitates the determination of potential regions with underdosage in a tumor due to hypoxia. By further implementing the method in a treatment planning tool, it will be possible to adapt prescription doses based on the local tumor oxygen level, also accounting for RBE effects through coupling with existing RBE models. The resulting biologically weighted dose at a hypoxic condition compared to that in a normoxic condition was lower by a factor similar to the OER. For the head and neck cancer patient, this resulted in large areas of underdosage compared to the prescribed dose. While variable normoxic RBE models generally estimate higher biologically weighted dose than the $D_{\mathrm{RBE} 1.1}$, areas of underdosage were also observed when applying the $D_{\mathrm{ROR}}$ model adapted for hypoxia in biologically weighted dose estimations. The results in this study confirm that neglecting the effect of hypoxia in proton therapy potentially could compromise the expected tumor control probability and should therefore be kept in mind in clinical practice.

\section{Acknowledgments}

This work was partly funded by the Trond Mohn Foundation (funding no. BFS2015TMT03 and BFS2017TMT07) and the Norwegian Cancer Society (grant no. 182672-2016).

\section{Appendix A-D. Supplementary data}

Supplementary data to this article can be found online at https:// doi.org/10.1016/j.ejmp.2020.07.003.

\section{References}

[1] Carreau A, El Hafny-Rahbi B, Matejuk A, Grillon C, Kieda C. Why is the partial oxygen pressure of human tissues a crucial parameter? Small molecules and hypoxia. J Cell Mol Med 2011;15:1239-53. https://doi.org/10.1111/j.1582-4934. 2011.01258.x.

[2] Furusawa Y, Fukutsu K, Aoki M, Itsukaichi H, Ohara H, Yatagai F, et al. Inactivation of Aerobic and Hypoxic Cells from Three Different Cell Lines by Accelerated $3 \mathrm{He}-$, 12C- and 20Ne-ion Beams 2000;154:485-96. https://doi.org/https://doi.org/10. 1667/0033-7587(2000)154[0485:IOAAHC]2.0.CO;2.

[3] ICRU. Prescribing recording, and reporting proton-beam therapy (ICRU Report 78). Int Comm Radiat Units Meas 2007.

[4] Wedenberg M, Lind BK, Hårdemark B. A model for the relative biological effectiveness of protons: the tissue specific parameter $\alpha / \beta$ of photons is a predictor for the sensitivity to LET changes. Acta Oncol 2013;52:580-8. https://doi.org/10. 3109/0284186X.2012.705892.

[5] Rørvik E, Thornqvist S, Stokkevåg CH, Dahle TJ, Fjæra LF, Ytre-Hauge KS. A phenomenological biological dose model for proton therapy based on linear energy transfer spectra. Med Phys 2017;44:2586-94. https://doi.org/10.1002/mp.12216.

[6] McNamara AL, Schuemann J, Paganetti H. A phenomenological relative biological effectiveness (RBE) model for proton therapy based on all published in vitro cell survival data. Phys Med Biol 2015;60:8399-416. https://doi.org/10.1088/00319155/60/21/8399.

[7] Carabe A, Moteabbed M, Depauw N, Schuemann J, Paganetti H. Range uncertainty in proton therapy due to variable biological effectiveness. Phys Med Biol 2012;57:1159-72. https://doi.org/10.1088/0031-9155/57/5/1159.

[8] Allemann K, Wyss MT, Wergin M, Ohlerth S, Rohrer-Bley C, Evans SM, et al. Measurements of hypoxia ([(18)F]-FMISO, [(18)F]-EF5) with positron emission tomography (PET) and perfusion using PET ([(15)O]-H(2)O) and power Doppler ultrasonography in feline fibrosarcomas*. Vet Comp Oncol 2005;3:211-21. https:// doi.org/10.1111/j.1476-5810.2005.00081.x.

[9] Chirla R, Marcu LG. PET-based quantification of statistical properties of hypoxic tumor subvolumes in head and neck cancer. Phys Medica 2016;32:23-35. https:// doi.org/10.1016/j.ejmp.2015.12.006.

[10] Fleming IN, Manavaki R, Blower PJ, West C, Williams KJ, Harris AL, et al. Imaging tumour hypoxia with positron emission tomography. Br J Cancer 2015;112:238-50. https://doi.org/10.1038/bjc.2014.610.

[11] Koch CJ, Evans SM. Optimizing hypoxia detection and treatment strategies. Semin Nucl Med 2015;45:163-76. https://doi.org/10.1053/j.semnuclmed.2014.10.004.

[12] Koch CJ. Measurement of absolute oxygen levels in cells and tissues using oxygen sensors and 2-nitroimidazole EF5. Methods Enzymol 2002;352:3-31. https://doi. org/10.1016/S0076-6879(02)52003-6.

[13] Komar G, Seppanen M, Eskola O, Lindholm P, Gronroos TJ, Forsback S, et al. 18FEF5: A New PET tracer for imaging hypoxia in head and neck cancer. J Nucl Med 2008;49:1944-51. https://doi.org/10.2967/jnumed.108.053785.

[14] Evans SM, Du KL, Chalian AA, Mick R, Zhang PJ, Hahn SM, et al. Patterns and levels 
of hypoxia in head and neck squamous cell carcinomas and their relationship to patient outcome. Int J Radiat Oncol Biol Phys 2009;69:1024-31. https://doi.org/ 10.1016/j.ijrobp.2007.04.067.

[15] Komar G, Lehtiö K, Seppänen M, Eskola O, Levola H, Lindholm P, et al. Prognostic value of tumour blood flow, $\left[{ }^{18} \mathrm{~F}\right] \mathrm{EF} 5$ and $\left[{ }^{18} \mathrm{~F}\right] \mathrm{FDG}$ PET/CT imaging in patients with head and neck cancer treated with radiochemotherapy. Eur J Nucl Med Mol Imag 2014;41:2042-50. https://doi.org/10.1007/s00259-014-2818-3.

[16] Böhlen TT, Cerutti F, Chin MPWW, Fassò A, Ferrari A, Ortega PG, et al. The FLUKA Code: Developments and challenges for high energy and medical applications. Nucl Data Sheets 2014;120:211-4. https://doi.org/10.1016/j.nds.2014.07.049.

[17] Ferrari A, Sala R, Fasso A, Ranft J. FLUKA: A multi-particle transport code. INFN/TC 2005;5:31-49. https://doi.org/10.2172/877507.

[18] Scholz M, Kraft G. Track structure and the calculation of biological effects of heavy charged particles. Adv Sp Res 1996;18:5-14. https://doi.org/10.1016/02731177(95)00784-C.

[19] Mairani A, Brons S, Cerutti F, Fassò A, Ferrari A, Krämer M, et al. The FLUKA Monte Carlo code coupled with the local effect model for biological calculations in carbon ion therapy. Phys Med Biol 2010;55:4273-89. https://doi.org/10.1088/0031 9155/55/15/006.

[20] Inaniwa T, Furukawa T, Kase Y, Matsufuji N, Toshito T, Matsumoto Y, et al. Treatment planning for a scanned carbon beam with a modified microdosimetric kinetic model. Phys Med Biol 2010;55:6721-37. https://doi.org/10.1088/0031 9155/55/22/008

[21] Magro G, Dahle TJ, Molinelli S, Ciocco M, Fossati P, Ferrari A, et al. The FLUKA Monte Carlo code coupled with the NIRS approach for clinical dose calculations in carbon ion therapy. Phys Med Biol 2017;62:3814-27. https://doi.org/10.1088/ 1361-6560/aa642b.

[22] Bopp C, Hirayama R, Inaniwa T, Kitagawa A, Noda K. Adaptation of the microdosimetric kinetic model to hypoxia. Phys Med Biol 2016;61:7586-99. https://doi. org/10.1088/0031-9155/61/21/7586.

[23] Scifoni E, Tinganelli W, Weyrather WK, Durante M, Maier A, Krämer M. Including oxygen enhancement ratio in ion beam treatment planning: Model implementation and experimental verification. Phys Med Biol 2013;58:3871-95. https://doi.org/ 10.1088/0031-9155/58/11/3871.

[24] Strigari L, Torriani F, Manganaro L, Inaniwa T, Dalmasso F, Cirio R, et al. Tumour control in ion beam radiotherapy with different ions in the presence of hypoxia: An oxygen enhancement ratio model based on the microdosimetric kinetic model. Phys Med Biol 2018:63. https://doi.org/10.1088/1361-6560/aa89ae.

[25] Tinganelli W, Durante M, Hirayama R, Krämer M, Maier A, Kraft-Weyrather W, et al. Kill-painting of hypoxic tumours in charged particle therapy. Sci Rep 2015;5:1-13. https://doi.org/10.1038/srep17016.

[26] Krämer M, Scholz M. Treatment planning for heavy-ion radiotherapy: calculation and optimization of biologically effective dose. Phys Med Biol 2000;45:3319-30. https://doi.org/10.1088/0031-9155/45/11/314.

[27] Krämer M, Jäkel O, Haberer T, Kraft G, Schardt D, Weber U. Treatment planning for heavy-ion radiotherapy: physical beam model and dose optimization. Phys Med Biol 2000;45:3299-317. https://doi.org/10.1088/0031-9155/45/11/313.

[28] Wenzl T, Wilkens JJ. Modelling of the oxygen enhancement ratio for ion beam radiation therapy. Phys Med Biol 2011;56:3251-68. https://doi.org/10.1088/ 0031-9155/56/11/006.

[29] Silvoniemi A, Suilamo S, Laitinen T, Forsback S, Löyttyniemi E, Vaittinen S, et al. Repeatability of tumour hypoxia imaging using [18F]EF5 PET/CT in head and neck cancer. Eur J Nucl Med Mol Imag 2018;45:161-9. https://doi.org/10.1007/s00259017-3857-3.

[30] Toma-Dasu I, Uhrdin J, Daşu A, Brahme A. Therapy, optimization based on nonlinear uptake of PET tracers versus "linear dose painting". IFMBE Proc
2009;25:221-4. https://doi.org/10.1007/978-3-642-03474-9_63.

[31] Kellerer AM, Rossi HH. A generalized formulation of dual radiation action. Radiat Res 1978;75:471-88. https://doi.org/10.2307/3574835.

[32] Rørvik E, Fjæra LF, Dahle TJ, Dale JE, Engeseth GM, Stokkevåg CH, et al Exploration and application of phenomenological RBE models for proton therapy. Phys Med Biol 2018;63:185013https://doi.org/10.1088/1361-6560/aad9db.

[33] van Leeuwen CM, Oei AL, Crezee J, Bel A, Franken NAP, Stalpers LJA, et al. The alfa and beta of tumours: A review of parameters of the linear-quadratic model, derived from clinical radiotherapy studies. Radiat Oncol 2018;13:1-11. https://doi.org/10. 1186/s13014-018-1040-Z

[34] Fjæra LF, Li Z, Ytre-Hauge KS, Muren LP, Indelicato DJ, Lassen-Ramshad Y, et al. Linear energy transfer distributions in the brainstem depending on tumour location in intensity-modulated proton therapy of paediatric cancer. Acta Oncol (Madr) 2017;56:763-8. https://doi.org/10.1080/0284186X.2017.1314007.

[35] Wouters BG, Brown JM. Cells at Intermediate Oxygen Levels Can Be More Important Than the "Hypoxic Fraction" in Determining Tumor Response to Fractionated Radiotherapy. Radiat Res 1997;147:541. https://doi.org/10.2307/ 3579620.

[36] Malinen E, Søvik Å, Hristov D, Bruland ØS, Olsen DR. Adapting radiotherapy to hypoxic tumours. Phys Med Biol 2006;51:4903-21. https://doi.org/10.1088/0031 9155/51/19/012

[37] Antonovic L, Brahme A, Furusawa Y. Radiobiological description of the LET dependence of the cell survival of oxic and anoxic cells irradiated by carbon ions. $\mathrm{J}$ Radiat Res 2013;54:18-26. https://doi.org/10.1093/jrr/rrs070.

[38] Dahle TJ, Rykkelid AM, Stokkevåg CH, Mairani A, Görgen A, Edin NJ, et al. Monte Carlo simulations of a low energy proton beamline for radiobiological experiments Acta Oncol (Madr) 2017;56:779-86. https://doi.org/10.1080/0284186X.2017. 1289239.

[39] Durante M, Paganetti H, Pompos A, Kry SF, Wu X, Grosshans DR. Report of a National Cancer Institute special panel: Characterization of the physical parameters of particle beams for biological research. Med Phys 2019;46:e37-52. https://doi org/10.1002/mp.13324.

[40] Wenzl T, Wilkens JJ. Theoretical analysis of the dose dependence of the oxygen enhancement ratio and its relevance for clinical applications. Radiat Oncol 2011;6:171. https://doi.org/10.1186/1748-717X-6-171.

[41] Kjellsson Lindblom E, Ureba A, Dasu A, Wersäll P, Even AJG, van Elmpt W, et al. Impact of SBRT fractionation in hypoxia dose painting - Accounting for heterogeneous and dynamic tumor oxygenation. Med Phys 2019;46:2512-21. https://doi. org/10.1002/mp.13514

[42] Sokol O, Krämer M, Hild S, Durante M, Scifoni E. Kill painting of hypoxic tumors with multiple ion beams. Phys Med Biol 2019;64. https://doi.org/10.1088/13616560/aafe40.

[43] Malinen E, Søvik Å. Dose or LET painting - What is optimal in particle therapy of hypoxic tumors? Acta Oncol (Madr) 2015;54:1614-22. https://doi.org/10.3109/ 0284186X.2015.1062540.

[44] Bassler N, Toftegaard J, Lühr A, Sørensen BS, Scifoni E, Krämer M, et al. LETpainting increases tumour control probability in hypoxic tumours. Acta Oncol (Madr) 2014;53:25-32. https://doi.org/10.3109/0284186X.2013.832835.

[45] Forster JC, Marcu LG, Bezak E. Approaches to combat hypoxia in cancer therapy and the potential for in silico models in their evaluation. Phys Medica 2019;64:145-56. https://doi.org/10.1016/j.ejmp.2019.07.006.

[46] Chang JH, Wada M, Anderson NJ, Lim Joon D, Lee ST, Gong SJ, et al. Hypoxiatargeted radiotherapy dose painting for head and neck cancer using18F-FMISO PET: A biological modeling study. Acta Oncol (Madr) 2013;52:1723-9. https://doi.org/ 10.3109/0284186X.2012.759273. 